\title{
Microscopic Findings Measurement, Test or Examination Detail
}

National Cancer Institute

\section{Source}

National Cancer Institute. Microscopic Findings Measurement, Test or Examination

Detail. NCI Thesaurus. Code C117579.

Particulars considered individually and in relation to a microscopic findings measurement, test or examination. 